\title{
Multi-spacecraft observations of heavy-ion solar energetic particles
}

\author{
Peter Zelina* \\ University of Central Lancashire, Preston, United Kingdom \\ E-mail: pzelina@uclan.ac.uk

\section{Silvia Dalla} \\ University of Central Lancashire, Preston, United Kingdom \\ E-mail: sdalla@uclan.ac.uk

\section{Timo Laitinen} \\ University of Central Lancashire, Preston, United Kingdom \\ E-mail: tlmlaitinen@uclan.ac.uk
}

\begin{abstract}
Solar Energetic Particles (SEPs) released during flares and coronal mass ejections can be detected by spacecraft widely separated in longitude, and the mechanism by which this takes place remains unclear. Studies of SEP events simultaneously detected by multiple spacecraft have mostly focussed on electron and proton data. Here we consider multi-spacecraft events observed by the LET instrument on STEREO A and B, and by SIS on board ACE located near Earth, and analyse the properties of heavy ion SEPs. We study the intensity time profiles of $\mathrm{Fe} / \mathrm{O}$ ratio in six SEP events observed between December 2006 and September 2014. The results show that decreases of $\mathrm{Fe} / \mathrm{O}$ in time are common features of SEP events. Observed decreases and mean $\mathrm{Fe} / \mathrm{O}$ values vary considerably from event to event.
\end{abstract}

The 34th International Cosmic Ray Conference

30 July-6 August, 2015

The Hague, The Netherlands

${ }^{*}$ Speaker. 


\section{Introduction}

Solar flares and coronal mass ejections (CMEs) can accelerate charged particles to high kinetic energies. These ions and electrons are known as solar energetic particles (SEPs). SEP events are often divided into two classes known as gradual and impulsive events [1], based mainly on ${ }^{3} \mathrm{He} /{ }^{4} \mathrm{He}$ and $\mathrm{Fe} / \mathrm{O}$ ratio abundances. Gradual SEP events, understood to be caused by a CME-driven shock, cause the largest proton intensity increases. Observed SEP abundances in gradual events are similar to spectroscopic observations in the solar corona [2,3]. Values of ${ }^{3} \mathrm{He} /{ }^{4} \mathrm{He}$ and $\mathrm{Fe} / \mathrm{O}$ ratios detected during impulsive SEP events are often increased by several orders of magnitude above the average SEP coronal abundances [1].

The current view on the propagation of SEPs is that they travel along magnetic field lines without much perpendicular transport [1]. In gradual events, the width of the expanding shock is thought to be responsible for the wide extent of detected SEPs. On the other hand, SEPs observed during impulsive events would only be detected in narrow regions with good magnetic connection. However, this is contradicted by recent STEREO observations [4].

Observations of SEP events detected simultaneously by 2 or 3 spacecraft show that SEPs can reach regions with poor magnetic connection to the solar source region of the event, e.g. [5, 6]. In April 2013, the STEREO B and ACE spacecraft detected an iron rich SEP event at 2 widely separated locations [7]. Another report of heavy ion SEPs simultaneously observed by WIND and ULYSSES was made by [8]. In some cases, SEPs are thought to fill in the entire heliosphere [9]. These studies suggest there exists an effective transport mechanism that can distribute SEPs up to Fe to regions with poor magnetic connection. Hence, detection of SEPs by multiple spacecraft is desirable for understanding SEP propagation in the heliosphere.

The Fe/O abundance ratio has been historically the most commonly studied heavy ion ratio. It has been used for impulsive/gradual event classification $[1,4,10]$ and SEP transport and longitudinal dependence analyses $[7,8,9,11,12]$. The typical event-averaged $\mathrm{Fe} / \mathrm{O}$ abundance ratio in gradual SEP events is $A_{\mathrm{Fe} / \mathrm{O}} \equiv I_{\mathrm{Fe}} / I_{\mathrm{O}}=0.134$ at $5-12 \mathrm{MeV} /$ nuc. [2].

Past works have shown that during an SEP event an initially high $\mathrm{Fe} / \mathrm{O}$ ratio can decrease in time to the average gradual $\mathrm{Fe} / \mathrm{O}$ abundance. The time dependence of $\mathrm{Fe} / \mathrm{O}$ reported by [13] was interpreted as an effect of a rigidity-dependent mean free path. Similar behaviour in Fe/O time profiles was reported by [14], who suggested that the features of acceleration at a CME driven shock and particle escape from it were responsible [15]. Mason et al. [12] analysed 17 SEP events, where temporal variation of Fe/O was observed at $276 \mathrm{keV} /$ nuc. and $12 \mathrm{MeV} /$ nuc. (high energies being relevant to this study). They concluded that Fe/O decreases could be better understood as a transport effect related to the rigidity dependence of the scattering mean free path. Tylka et al. [8] used multiple spacecraft in the ecliptic plane (WIND) and at high heliolatitudes (ULYSSES), with various magnetic connectivity to the parent eruptive event. They came to a similar conclusion as [12] that the initial $\mathrm{Fe} / \mathrm{O}$ enhancement was more likely to be explained by a rigidity-dependent transport process. Another possible mechanism proposed to explain the observed initially high values of $\mathrm{Fe} / \mathrm{O}$ is a dual source for SEPs with an initial Fe-rich flare component [16], however, this scenario is generally incompatible with those mentioned above.

Both the analytical expressions for charged particle drifts of SEPs [17] and full-orbit simulations of SEPs in the Parker spiral [18] suggest that SEP drifts are an important mechanism in the 
perpendicular transport, and can effectively distribute SEPs across the interplanetary magnetic field. The main contributors to the drifts are the gradient B drift and the curvature drift. The drifts depend on $m / q$ and kinetic energy, potentially fulfilling the requirement of rigidity-dependent mechanism. Simulations by Dalla et al. [19] show that drift processes can indeed produce time profiles of Fe/O decreasing in time.

In this analysis, we focus on the time evolution of $\mathrm{Fe} / \mathrm{O}$ as measured in six events between 2006 and 2014. Observed Fe/O abundances show initially increased values at the beginning of SEP events decaying with time. Moreover, we find that heavy ions up to Fe can be detected at spacecraft widely separated in longitude similarly to protons and electrons $[5,6]$. We aim to describe $\mathrm{Fe} / \mathrm{O}$ decreases and $\mathrm{Fe} / \mathrm{O}$ mean values in these events and discuss how this could be a signature of a transport effect.

\section{Data and instrumentation}

The datasets used in the analysis comprise heavy ion SEP intensity data measured by the Solar Isotope Spectrometer (SIS) [20] onboard the Advanced Composition Explorer (ACE), and the Low Energy Telescope (LET) [21] onboard the Solar TErrestrial RElations Observatory (STEREO) Ahead and Behind. Level 2 SEP data are maintained and released by the instrument teams and they are publicly available. The kinetic energy of SEPs is measured by stacked silicon detectors using the energy loss technique. SEP data are organised into differential energy channels in the energy range $\approx 4-60 \mathrm{MeV} /$ nuc.

We analyse six large solar eruptive events, each caused by a single parent eruptive event taking place between December 2006 and September 2014. Except for event \#1, at the time of the SEP events the ACE and STEREO spacecraft were at large longitudinal separation from each other (e.g. see Figure 1 for event \#6). In these events the measured intensities of $\mathrm{Fe}$ and $\mathrm{O}$ exceeded $10^{-3}\left(\mathrm{~cm}^{2} \mathrm{~s} \mathrm{srMeV} / \mathrm{nuc}\right)^{-1}$ at $10-12 \mathrm{MeV} /$ nuc. in STEREO/LET data. The threshold for $\mathrm{ACE} / \mathrm{SIS}$ was $10^{-4}\left(\mathrm{~cm}^{2} \mathrm{~s} \mathrm{srMeV} / \mathrm{nuc}\right)^{-1}$ due to its higher detector collection area. We also require good counting statistics, where only $>2$ particles/time bin are included in the analysis. Details of the selected SEP events are listed in Table 1. The event-averaged mean Fe/O value is calculated as the mean of the ratio over the event duration.

\section{Results}

Plots of $\mathrm{O}$ and $\mathrm{Fe}$ intensity (left) and $\mathrm{Fe} / \mathrm{O}$ ratio (right) versus time at the best magnetically connected spacecraft are shown in Figure 2. Energy channels used in the analysis are $4.0-4.5 \mathrm{MeV} /$ nuc. and $10-15 \mathrm{MeV} /$ nuc. for STEREO/LET and ACE/SIS, respectively.

For all events, the ratio plots show decreases of $\mathrm{Fe} / \mathrm{O}$ over time present in the data. Initially enhanced $\mathrm{Fe} / \mathrm{O}$ abundance ratios decrease to values similar to the average $\mathrm{Fe} / \mathrm{O}$ abundance for gradual events of 0.134 . The decreases take 1 to 2 days after the SEP event onset and vary between $\approx 0.5$ and 1.5 orders of magnitude. Events \#1 and \#5 have mean Fe/O several times above the standard gradual SEP average value, while the other events' mean Fe/O are close to or below 0.134. The observed initial enhancements may be responsible for the calculated high mean $\mathrm{Fe} / \mathrm{O}$ values. 


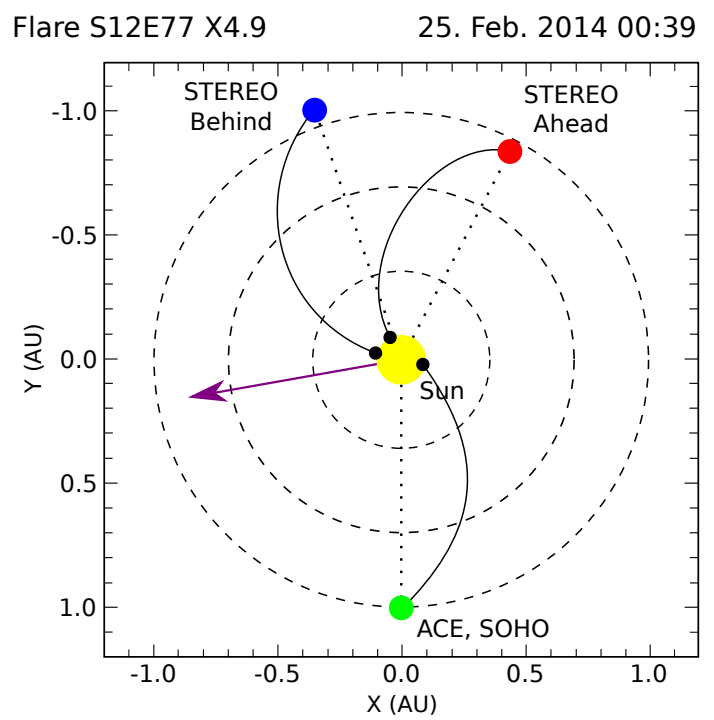

Figure 1: Position of ACE, STEREO A and B in the ecliptic plane at the onset of event \#6. The arrow marks the radial directionality of the flare.

Table 1: Details of selected SEP events.

\begin{tabular}{rcccllcr}
\hline$\#$ & Year & Duration (day) & Flare peak $^{\dagger}$ & Flare class $^{\dagger}$ & Location $^{\dagger}$ & Mag. connection & CME speed $^{\ddagger}\left[\mathrm{km}^{\text {s }}\right]$ \\
\hline 1 & 2006 & $347-349$ & Dec 13/0240 & X3.4 & S05W23 & $-27^{\circ}$; ACE & 1774 \\
2 & 2011 & $216-219$ & Aug 04/0412 & M9.3 & N15W49 & $-10^{\circ}$; ACE & 1610 \\
3 & 2012 & $244-248$ & Aug 31/2043 & C8 $^{b}$ & S16E42 & $-5^{\circ}$; STB & 1442 \\
4 & 2013 & $64-67$ & Mar 05/0316 & NI $^{\sharp}$ & NI & NI $^{\sharp}$ STA & 1316 \\
5 & 2013 & $101-104$ & Apr 11/0716 & M6.5 & N07E13 & $56^{\circ}$; STB & 861 \\
6 & 2014 & $56-59$ & Feb 25/0049 & X4.9 & S12E77 & $32^{\circ}$; STB & 2147 \\
\hline
\end{tabular}

${ }^{\dagger}$ from SolarMonitor.org [22]; $¥ \mathrm{CDAW}$ catalogue [23]; ${ }^{b}$ filament eruption; $\sharp$ NI = not identified, backside flare $\mathrm{STB}=$ STEREO B; STA $=$ STEREO A

Figure 3 shows $\mathrm{Fe}$ and $\mathrm{O}$ intensities (left) and Fe/O ratio (right) at STEREO B, ACE, and STEREO A (top to bottom) during the initial part of event \#6. The SEP event related to a large eruptive event (flare class X4.9 [22], CME speed $2147 \mathrm{~km} / \mathrm{s}$ [23]) caused both Fe and O ions to be detected at all three spacecraft widely separated in longitude. The longitudinal separation $\Delta \phi$ $\left(=\phi_{\text {flare }}-\phi_{\text {footpoint }}\right)$ between the parent active region and magnetic footpoints of STEREO B, ACE and STEREO A are $32^{\circ},-131^{\circ}$ and $70^{\circ}$, respectively. STEREO B is the best magnetically connected spacecraft to the X4.9 flare, followed by STEREO A and ACE. The measured peak intensities of $\mathrm{Fe}$ and $\mathrm{O}$ follow this pattern.

In contrast with the large event-to-event variation of mean $\mathrm{Fe} / \mathrm{O}$ in the events \#1-\#5 that can be seen in Figure 2, the mean $\mathrm{Fe} / \mathrm{O}$ at the three spacecraft during event $\# 6$ are similar to each other. 

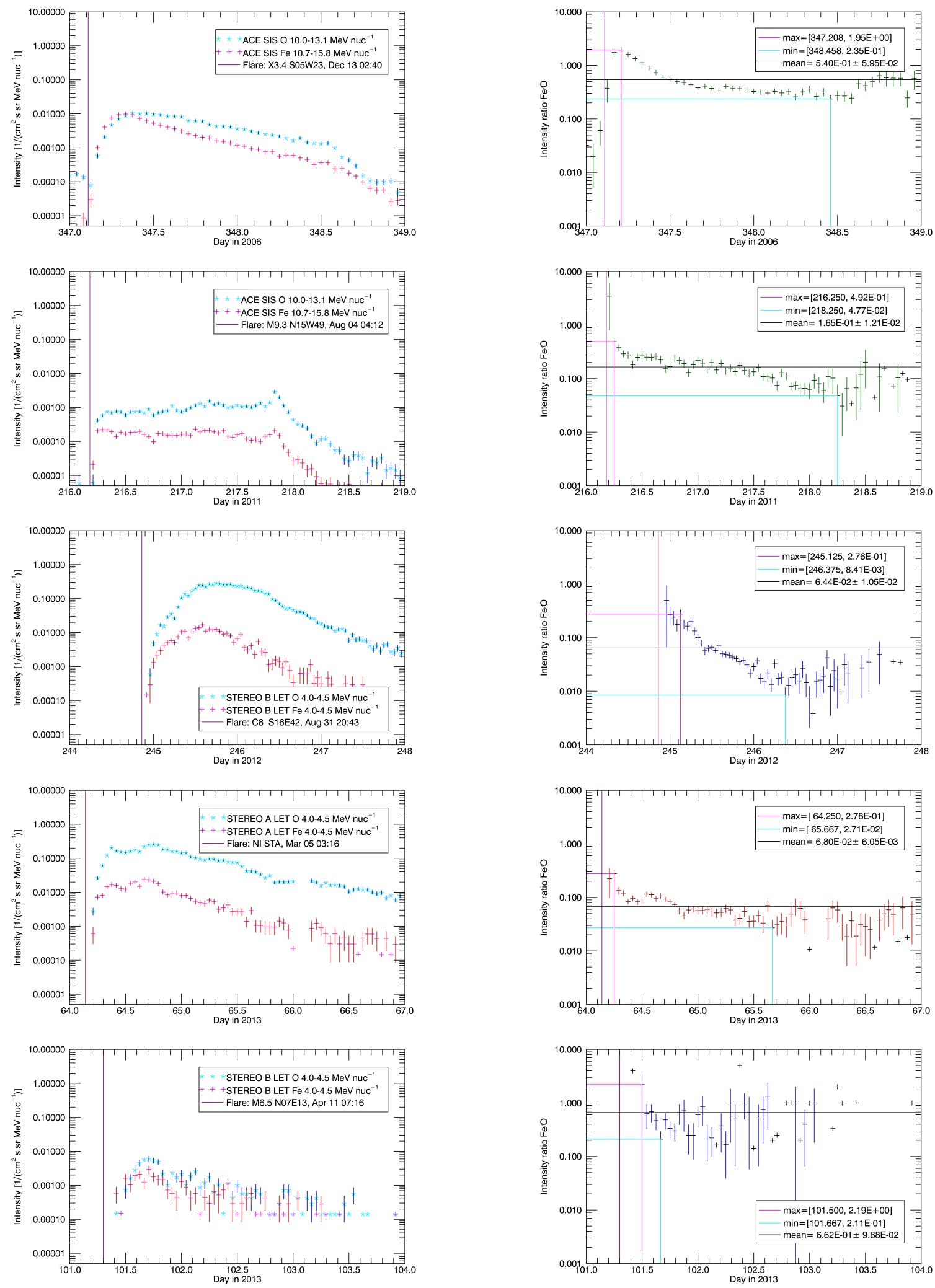

Figure 2: Fe (magenta) and $\mathrm{O}$ (cyan) intensity plots (left) and their ratio (right) time profiles during SEP events \#1 to \#5 at the best magnetically connected spacecraft. Purple vertical line indicates peak time of flare. Black horizontal line in the ratio plot shows mean $\mathrm{Fe} / \mathrm{O}$ value. 
Time profiles of $\mathrm{Fe} / \mathrm{O}$ of event \#6 also show less variation between the maxima and minima than in the events of Figure 2.
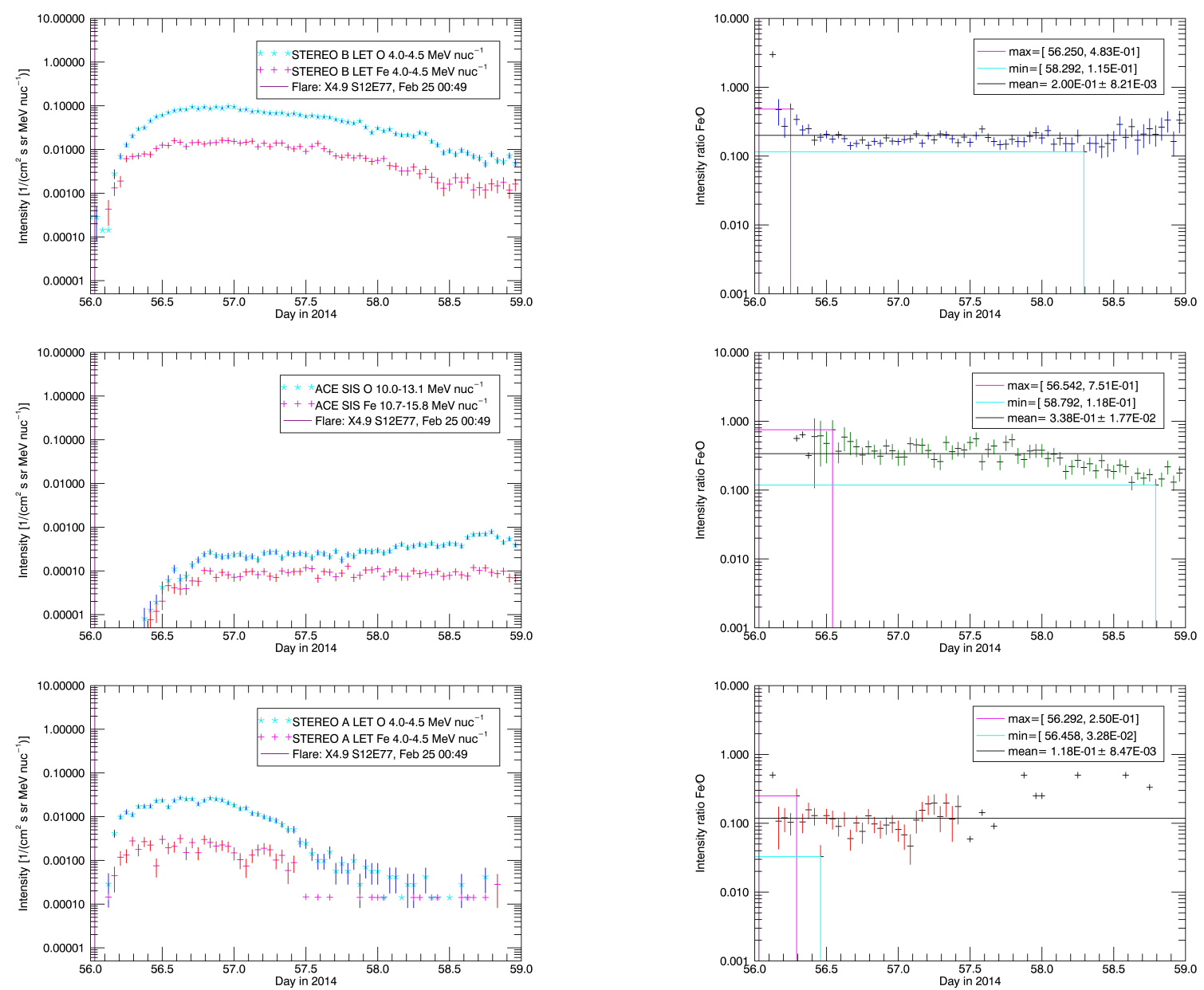

Figure 3: $\mathrm{Fe}$ (magenta) and $\mathrm{O}$ (cyan) intensity plots (left) and their ratio (right) time profiles during SEP event \#6 at STEREO B, ACE and STEREO A.

\section{Conclusions}

We analysed $\mathrm{Fe}$ and $\mathrm{O}$ intensities and their ratios at multiple spacecraft during six SEP events. All SEP events show $\mathrm{Fe} / \mathrm{O}$ decreasing in time after the event onset with high statistical confidence. The data show that a decrease of Fe/O ratio in time is a common feature of large SEP events rather than a phenomenon that may or may not be present. Our work represents the first study of this behaviour over a number of events at multiple spacecraft as opposed to case studies of one or 2 events.

Future work will focus on characterising the features of the Fe/O decay to verify if this allows identification of the mechanism responsible for the observed time dependence. 


\section{Acknowledgments}

This work has received funding from the UK Science and Technology Facilities Council (STFC) (grant ST/M00760X/1). Authors would like to thank the ACE SIS and STEREO LET instrument teams and the ACE and STEREO Science Centers for maintaining SEP data and making data publicly available.

\section{References}

[1] D. V. Reames, Particle acceleration at the Sun and in the heliosphere, Space Science Reviews 90 (1999) 413-491.

[2] D. Reames, Coronal abundances determined from energetic particles, Advances in Space Research 15 (1995) 41-51.

[3] D. V. Reames, Solar energetic particles: Sampling coronal abundances, Space Science Reviews 85 (1998) 327-340.

[4] M. E. Wiedenbeck, G. M. Mason, C. M. S. Cohen, N. V. Nitta, R. Gómez-Herrero, and D. K. Haggerty, Observations of solar energetic particles from ${ }^{3} \mathrm{He}$-rich events over a wide range of heliographic longitude, The Astrophysical Journal 762 (2013) 54.

[5] N. Dresing, Gómez-Herrero, R., Heber, B., Klassen, A., Malandraki, O., Dröge, W., and Kartavykh, Y., Statistical survey of widely spread out solar electron events observed with STEREO and ACE with special attention to anisotropies, Astronomy and Astrophysics 567 (2014) A27.

[6] I. Richardson, T. von Rosenvinge, H. Cane, E. Christian, C. Cohen, A. Labrador, R. Leske, R. Mewaldt, M. Wiedenbeck, and E. Stone, > $25 \mathrm{MeV}$ proton events observed by the High Energy Telescopes on the STEREO A and B spacecraft and/or at Earth during the first $\sim$ seven years of the STEREO mission, Solar Physics 289 (2014) 3059-3107.

[7] C. M. S. Cohen, G. M. Mason, R. A. Mewaldt, and M. E. Wiedenbeck, The longitudinal dependence of heavy-ion composition in the 2013 April 11 solar energetic particle event, The Astrophysical Journal 793 (2014) 35.

[8] A. J. Tylka, O. E. Malandraki, G. Dorrian, Y.-K. Ko, R. G. Marsden, C. K. Ng, and C. Tranquille, Initial Fe/O enhancements in large, gradual, solar energetic particle events: Observations from Wind and Ulysses, Solar Physics 285 (2013) 251-267.

[9] R. Gómez-Herrero, N. Dresing, A. Klassen, B. Heber, D. Lario, N. Agueda, O. E. Malandraki, J. Blanco, J. Rodríguez-Pacheco, and S. Banjac, Circumsolar energetic particle distribution on 2011 November 3, The Astrophysical Journal 799 (2015) 55.

[10] G. Wibberenz and H. V. Cane, Multi-spacecraft observations of solar flare particles in the inner heliosphere, The Astrophysical Journal 650 (2006) 1199.

[11] G. M. Mason, M. I. Desai, C. M. S. Cohen, R. A. Mewaldt, E. C. Stone, and J. R. Dwyer, The role of interplanetary scattering in western hemisphere large solar energetic particle events, The Astrophysical Journal Letters 647 (2006) L65-L68.

[12] G. M. Mason, G. Li, C. M. S. Cohen, M. I. Desai, D. K. Haggerty, R. A. Leske, R. A. Mewaldt, and G. P. Zank, Interplanetary propagation of solar energetic particle heavy ions observed at $1 \mathrm{AU}$ and the role of energy scaling, The Astrophysical Journal 761 (2012) 104. 
[13] M. Scholer, D. Hovestadt, B. Klecker, G. Gloeckler, and C. Y. Fan, Temporal development of the energetic particle composition during solar flares, Journal of Geophysical Research 83 (1978) 3349-3354.

[14] A. J. Tylka, D. V. Reames, and C. K. Ng, Observations of systematic temporal evolution in elemental composition during gradual solar energetic particle events, Geophysical Research Letters 26 (1999) 2141-2144.

[15] C. K. Ng, D. V. Reames, and A. J. Tylka, Effect of proton-amplified waves on the evolution of composition in gradual solar energetic particle events, Geophysical Research Letters 26 (1999) 2145-2148.

[16] H. V. Cane, T. T. von Rosenvinge, C. M. S. Cohen, and R. A. Mewaldt, Two components in major solar particle events, Geophysical Research Letters 30 (2003) 8017.

[17] S. Dalla, M. Marsh, J. Kelly, and T. Laitinen, Solar energetic particle drifts in the Parker spiral, Journal of Geophysical Research: Space Physics 118 (2013) 5979-5985.

[18] M. S. Marsh, S. Dalla, J. Kelly, and T. Laitinen, Drift-induced perpendicular transport of solar energetic particles, The Astrophysical Journal 774 (2013) 4.

[19] S. Dalla, M. Marsh, P. Zelina, and T. Laitinen, 3D simulations of heliospheric propagation of heavy-ion solar energetic particles, in International Cosmic Ray Conference (A. van den Berg, ed.), Proceedings of Science, 2015.

[20] E. Stone, C. Cohen, W. Cook, A. Cummings, B. Gauld, B. Kecman, R. Leske, R. Mewaldt, M. Thayer, B. Dougherty, R. Grumm, B. Milliken, R. Radocinski, M. Wiedenbeck, E. Christian, S. Shuman, and T. von Rosenvinge, The Solar Isotope Spectrometer for the Advanced Composition Explorer, Space Science Reviews 86 (1998) 357-408.

[21] R. Mewaldt, C. Cohen, W. Cook, A. Cummings, A. Davis, S. Geier, B. Kecman, J. Klemic, A. Labrador, R. Leske, H. Miyasaka, V. Nguyen, R. Ogliore, E. Stone, R. Radocinski, M. Wiedenbeck, J. Hawk, S. Shuman, T. von Rosenvinge, and K. Wortman, The Low-Energy Telescope (LET) and SEP Central Electronics for the STEREO mission, Space Science Reviews 136 (2008) 285-362.

[22] P. T. Gallagher, Y. Moon, and H. Wang, Active-region monitoring and flare forecasting - I. data processing and first results, Solar Physics 209 (2002) 171-183.

[23] CDAW Data Center, SoHO LASCO CME Catalog, . http: //cdaw.gsfc.nasa.gov/CME_list/ Accessed 2th April 2015. 